\title{
Optical Calculation with Three Sources
}

\author{
Zhou Zhimin ${ }^{1,}$ a , Wang Cailing ${ }^{2, b}$, and Xiaoyan $\mathrm{Li}^{3, \mathrm{c}}$ \\ ${ }^{1}$ Science and Technology on ship and marine Test and Control Laboratory, Dalian, China. \\ ${ }^{2}$ Science and Technology on Underwater Test and Control Laboratory, Dalian, China. \\ ${ }^{3}$ College of Life Science and Technology of Dalian University Liaoning,Dalian,China \\ azzm@dlut.edu.cn , bw_cailing@163.com , ${ }^{c} 112731815 @ q q . c o m$
}

Keywords: optical beacon, three lamp-houses, blind zeros, layout of beacon.

Abstract: The optical beacon is an important position indicator in aviation, sea transportation and the sea lifesaving. As an optical indicator, the optical beacon has been applied in many fields With the need of the remote indication, the optical beacon with LED sources, owing to high brightness and luminous efficiency, is present. Based on the established mathematical model of the light beam, the layout of the beacon with three lamp-houses is calculated theoretically, and the optimal layout, the ranges and the sizes of the blind zeros of the beacon are discussed. The results provide a theoretical guide for the structure design of the beacon essentially, and the method can be referred to layout of other multi-sources beacon.

\section{Introduction}

As an optical indicator, the optical beacon has been applied in many fields, such as the aviation, maritime transportation's traffic, sea rescue and so on [1-3]. The range of optical beacon should be large enough to find the person with the beacon timely in the sea rescue. And the size of optical beacon must be small for easy carry. LED (Light Emitting Diode) has the advantages of small volume, low power consumption, high brightness, high luminous efficiency, energy saving and environmental protection, solid state encapsulation, vibration resistance, impact resistance and so on. The energy consumption of white LED is only $1 / 10$ of that the incandescent lamp, and $1 / 4$ of that the energy-saving lamp [4-8].

The light from beacon can reach every corner of the half sphere above optical beacon in order that the rescuers find the target. For example, the light intensity of the life jacket light isn't below $0.75 \mathrm{~cd}$ in each direction in half-sphere of the beacon[2,9-11].

The optical beacon in this paper consists of 3 LED sources at open angle of $120^{\circ}$. Based on the mathematical model of the LED light beam, the layout of the sources is design, also the coverage and blind area of the beacon is discussed.

\section{Mathematical model of light beam}

As shown in Fig. 1, beam given out by the directional source is equivalent to a cone at vertex angle $\beta$, taking no account of the diffraction. The three sources are regarded as three points, and the beams from the sources are regarded as cones with the same vertex, equal vertex angle and different axis. When the angle between the cone axis and reference plane of the beacon is equal, the light in half spherical surface above optical beacon is uniform.

As shown in Fig. 1, xoy plane coincides with the reference plane of optical beacon, and LED sources are seated in the coordinate origin. In the figure, $\alpha$ is the angle between cone axis of 
light and xoy plane; $\gamma$ is the angel between projecting lines of neighbor cone axis in plane xoy. The axis of three light beam are evenly in a conical surface, which is a straight line family comprised the lines from the origin form surface and at an angle $\alpha$ with the xoy plane. The axis of cone 1 is in plane xoz.

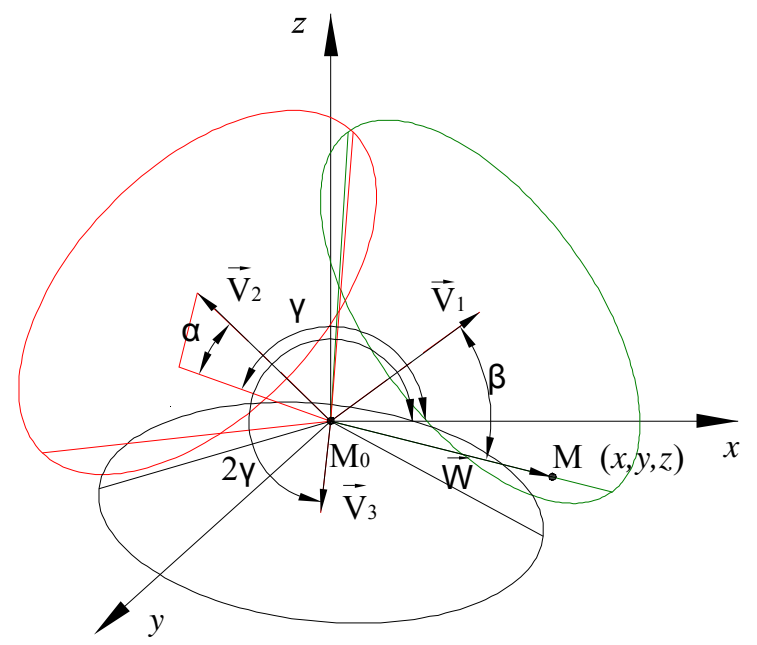

Fig. 1 Spatial distribution model of the three beam

The vector of the three axis $\stackrel{1}{V}_{1}, \stackrel{\prime}{V}_{2}, \stackrel{\prime}{V}_{3}$ is expressed as

$\stackrel{\prime}{V}_{1}=\{\cos \alpha, 0, \sin \alpha\}$

$\stackrel{\prime}{V}_{2}=\{\cos \gamma \cdot \cos \alpha, \sin \gamma \cdot \cos \alpha, \sin \alpha\}$

$\stackrel{\prime}{V}_{3}=\{\cos 2 \gamma \cdot \cos \alpha, \sin 2 \gamma \cdot \cos \alpha, \sin \alpha\}$

$M_{0}(0,0,0)$ is the vertex of the light cone; $M(x, y, z)$ is any point on the beam surface; The generating line of the cone surface is ${ }^{\prime} W=\{x, y, z\}$. The equation of the conical surface, which vertex point is $M_{0}$, axis is ${ }^{\prime}$, and vertex angle is $\beta$, can be expressed as

$$
\frac{\stackrel{v}{V} \cdot \stackrel{v}{W}}{|V| V|W|}=\cos \frac{\beta}{2}
$$

For the even distribution of the three axis in the lines family, we obtained $\gamma=120^{\circ}$ by the geometric relation. And the open angle $\beta=120^{\circ}$, the surfaces of the three light cones are formulated as

$$
\begin{aligned}
& (2 x \cos \alpha+2 z \sin \alpha)^{2}=x^{2}+y^{2}+z^{2} \\
& (-x \cos \alpha+\sqrt{3} y \cos \alpha+2 z \sin \alpha)^{2}=x^{2}+y^{2}+z^{2}
\end{aligned}
$$


$(-x \cos \alpha-\sqrt{3} y \cos \alpha+2 z \sin \alpha)^{2}=x^{2}+y^{2}+z^{2}$

\section{Coverage conditions, coverage and blind area of the optical beacon}

\section{Coverage conditions of the beacon}

The following conditions should be provided in order that light arrive each corner in half sphere above the conference plane of the beacon.

1) The public domain exists in three light cones.

2) The intersecting lines between the three surfaces of the light cone irradiating from the origin are in or below the plane xoy.

\section{Coverage and blind area of the beacon}

The surface equations of the three light cones sent from LED sources are expressed as Eqs.(5), (6) and (7), and any beam from optical beacon is in the cone. If the three cones can cover whole above xoy plane, light sent from the beacon can achieve every corner of the hemisphere above reference plane of the optical beacon. Otherwise uncovered area is the blind spot of the beacon. From Eqs(5), (6) and (7), intersecting curves between the surfaces are deduced as

$$
\left\{\begin{array} { r l } 
{ ( 2 x \operatorname { c o s } \alpha + 2 z \operatorname { s i n } \alpha ) ^ { 2 } } & { = x ^ { 2 } + y ^ { 2 } + z ^ { 2 } } \\
{ ( - x \operatorname { c o s } \alpha + \sqrt { 3 } y \operatorname { c o s } \alpha + 2 z \operatorname { s i n } \alpha ) ^ { 2 } } & { x ^ { 2 } + y ^ { 2 } + z ^ { 2 } }
\end{array} \quad \Rightarrow \left\{\begin{array}{l}
y=\sqrt{3} x \\
z=\frac{2 \sin \alpha}{2 \cos \alpha \pm \sqrt{3}} x
\end{array}\right.\right.
$$

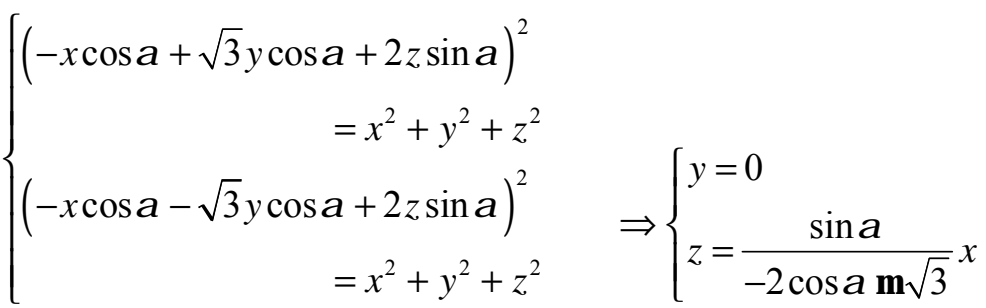

$$
\begin{aligned}
& \left\{\begin{array} { r l } 
{ ( - x \operatorname { c o s } \alpha - \sqrt { 3 } y \operatorname { c o s } \alpha + 2 z \operatorname { s i n } \alpha ) ^ { 2 } } & { } \\
{ = } & { x ^ { 2 } + y ^ { 2 } + z ^ { 2 } } \\
{ ( 2 x \operatorname { c o s } \alpha + 2 z \operatorname { s i n } \alpha ) ^ { 2 } = } & { x ^ { 2 } + y ^ { 2 } + z ^ { 2 } }
\end{array} \quad \Rightarrow \left\{\begin{array}{l}
y=-\sqrt{3} x \\
z=\frac{2 \sin \alpha}{2 \cos \alpha \pm \sqrt{3}} x
\end{array}\right.\right.
\end{aligned}
$$

In Eqs.(8), (9) and (10), for the equation $z=f(\alpha) x$, when the coefficient expression $f(\alpha)=0$, the intersecting lines are in plane xoy. Only when $\alpha=0, \mathrm{f}(\alpha)=0$, the intersecting lines between the surfaces are in plane xoy, also the axis of the cones are in plane xoy. But no public domain exists above the beacon, and the blind area is large, as shown in Fig. 2 (a). So there must be some blind area in the semi-sphere above the reference surface of the optical beacon with three LED sources at open angle $120^{\circ}$.

As shown in Fig. 2 (b), when $\alpha=90^{\circ}-\beta / 2=30^{\circ}$, there are four intersecting lines between the three cones, and minimum blind area is in the half spherical above the beacon. The lines OA, OB and OC are intersecting lines between the three cone surfaces. The points $\mathrm{M}, \mathrm{N}, \mathrm{S}, \mathrm{R}, \mathrm{K}$ and $\mathrm{L}$ are intersect points of lengthening the intersecting lines of the cone and the plane xoy respectively to the surface on sphere which $R=\mathrm{OA}$, in Fig. 2(b). The blind area of the beacon above xoy plane is composed 
of three same size areas OAMN, OBSR and OCKL. Supposed the small blind area is $\Omega$, the blind area of the beacon

$V=3 \iiint_{\Omega} d x d y d z$

When $\alpha=30^{\circ}, z=0$, the equations of intersecting line between three cone and xoy plane respectively are derived from Eqs. (5) - (7)

$$
\left\{\begin{array} { l } 
{ y = \pm \sqrt { 2 } x } \\
{ z = 0 }
\end{array} \quad \left\{\begin{array} { l } 
{ y = \frac { x } { - 3 \sqrt { 3 } \pm 4 \sqrt { 2 } } } \\
{ z = 0 }
\end{array} \quad \left\{\begin{array}{l}
y=\frac{x}{3 \sqrt{3} \pm 4 \sqrt{2}} \\
z=0
\end{array}\right.\right.\right.
$$

Two intersecting lines between a cone and the xoy plane are the generating lines of the cone. By Eq.(11), the included angle between the two intersecting lines on the same conical surface $\phi=2 \arctan \sqrt{2}=109.5^{\circ}$

The angle included in intersecting lines, between the adjacent cones and xoy plane, can be $\underset{\text { expressed as }}{\psi} \psi \angle \mathrm{MON}=\frac{360-3 \phi}{3}=120-\phi$.

The arc length between point $\mathrm{M}$ and $\mathrm{N}$ on the circle, intersecting curve between plane xoy and spherical surface with radius $\mathrm{R}$, is deduced as $\overline{\mathrm{MN}} \approx \pi \frac{\angle \mathrm{MON}}{180} R=\frac{\psi}{180} \pi R$.

By equation (9), the height of point $\mathrm{A}$ is obtained

$$
\mathrm{AA}^{\prime \prime}=\left|z_{0}\right|=\left|-\frac{\sqrt{3}}{12} x_{0}\right|
$$

$\triangle \mathrm{AMN}$ is an isosceles triangle due to the uniformity of three cones, and its high AA 'is in the plane OAA'. According the geometric relation in Fig. 2 (b), the height of $\triangle \mathrm{AMN}$ is obtained $\mathrm{H}=0.143 \mathrm{R}$.

The volume of the area $\Omega$ can be expressed $V_{z} \approx \frac{1}{6} \times H \times \overline{\mathrm{MN}} \times R$.

The volume of the half sphere with radius $\mathrm{R}$ is expressed $V_{q}=\frac{2}{3} \pi R^{3}$.

The percentage of the blind area of optical beacon with three sources $i=3 \frac{V_{z}}{V_{q}} \approx 0.6 \%$.

On the calculation above, it is shown that the blind area is smallest, and the percentage of it is only $0.6 \%$ when the sources distribute uniformly and their axis is at angle $30^{\circ}$ for the optical beacon with three LED sources at $120^{\circ}$ open angle.

\section{Simulation of optical beacon's beam}

The light beam model of the three sources is built by UG, as shown in Fig. 2. 


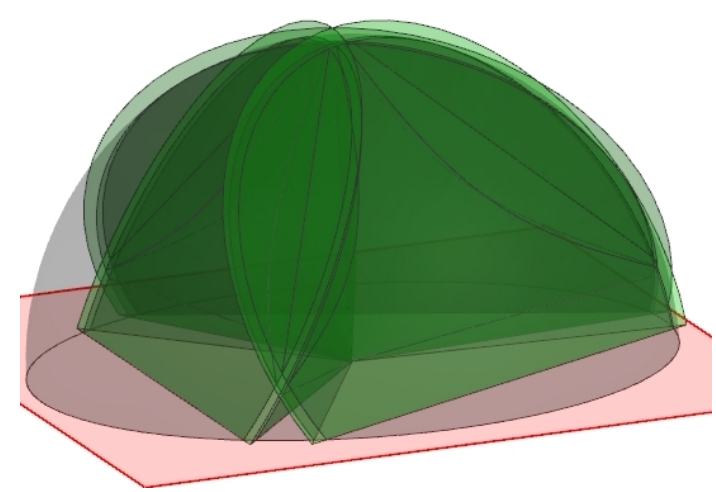

Fig.2 Three-dimensional simulation model $\alpha$ and area percentage $\mathrm{i}$

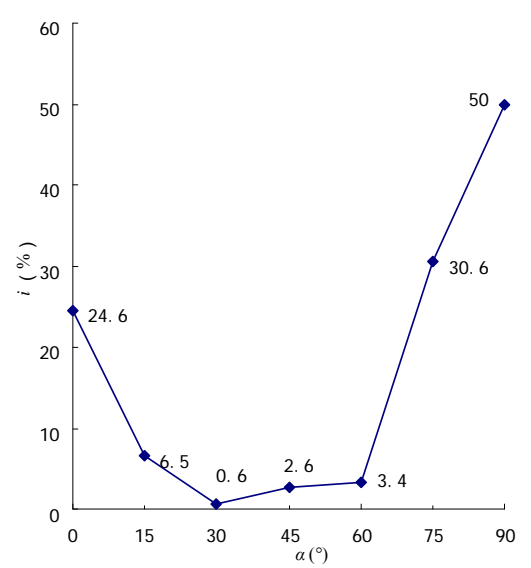

Fig.3 Relationship between axis angle

Blind area percentage varies with the angle of the beam axis $\alpha$, as shown in Fig. 3. With the increase of angle $\alpha$, the percentage decreases and then increases, and when $\alpha=30^{\circ}$, it reaches the minimum. On the analysis above, it is shown that the simulation and theoretical calculation results fit well.

When $\alpha=0^{\circ}$, the addition of a source with open angle less than 180 degree in the origin $\mathrm{O}$ cann't make the optical beacon cover the whole half sphere above the conference surface of it. For the three sources optical beacon, the blind area is only $0.6 \%$, when $\alpha=30^{\circ}$.

\section{Conclusions}

Taking no account of the light diffraction, the mathematical model and the simulation of the coverage of the optical beacon with three LED sources is presented, the results suggest that:

1) There must be some blind area in the half sphere above the conference surface of the optical beacon composed by three LED sources at open angle of $120^{\circ}$.

2) For the optical beacon composed by three LED sources at open angle of $120^{\circ}$, the blind area is only $0.6 \%$, when $\alpha=30^{\circ}$.

3) When $\alpha=0^{\circ}$, the addition of a source in the origin O, with open angle less than 180 degree, can't make the optical beacon cover the whole half sphere above the conference surface of it. In one words, light beacon with four LED sources can't cover the whole half sphere above the conference surface of the beacon also.

\section{Acknowledgement}

The study was supported by the National Natural Science Foundation of China (Grant No. 51349005 and 51375074 ) and by the "National Key Technology R\&D Program" in general program under grant No. 2013BAK03B06.

\section{References}

[1]Hans PoulAlkjaer,2011. Emergency Light Device for Marine Environments. United States Patent Application Publication. US 20110200331 AL, Sep. 1, 2011.

[2]Meng Xianlin,2002. Marine rescue Indicator of foreign country. Forum of China Personal Protection Equipment (CPPE), 2002, ( 6 ) : 24-25.

[3]Ding Yuanyi,2003. Lifesaving inspiration of the US marine. Symposium of Rescue and Salvage Committee, 2003:45-47. 
[4]Tu Hao,2013. An Optical Design Based on Improving LED Array Far Field Illumination Intensity. China Illuminating Engineering Journal, 2013, 24(1):74-80.

[5]Liu Yibing,2008. Research of Heat Release Technology of Based on Power-LED. China Illuminating Engineering Journal, 2008 , 19 (1):69-73.

[6]Dou Linping,2011. Discussion on LED Lighting Application in China. China Illuminating Engineering Journal, 2011, 22(6) :51-58.

[7]K.C. Yung,2014. Heat transfer analysis of a high-brightness LED array on PCB under different placement configurations. International Communications in Heat and Mass Transfer, 2014, 53: 79-86.

[8]Enguo Chen,2013. Design of LED-based reflector-array module for specific illuminance distribution. Optics Communications, 2013, 289:19-27.

[9]Xiaohong,2005. Structure and performance of the life jacket. Development Proceedings of Modern High-tech Textile. 2005:391-395.

[10] Wang Jun,2011. Standards and test method about position-indicating lights for life-saving appliances. Chinese Fishery Quality and Standards, 2011, 1(3) :51-58.

[11] GB5869-2010. 